\title{
Skin Flap Necrosis by Bone Marking with Methylene Blue in Cochlear Implantation
}

\author{
Yeon Hoo Kim and Sung II Cho \\ Department of Otolaryngology-Head and Neck Surgery, Chosun University School of Medicine, Gwangju, Korea
}

Received May 28, 2015

Revised July 14, 2015

Accepted July 20, 2015

\author{
Address for correspondence \\ Sung Il Cho, MD, PhD \\ Department of Otolaryngology- \\ Head and Neck Surgery, \\ Chosun University \\ School of Medicine, \\ 365 Pilmun-daero, \\ Dong-gu, Gwangju 61453, Korea \\ Tel $+82-62-220-3207$ \\ Fax +82-62-225-2702 \\ E-mail chosi@chosun.ac.kr
}

One of surgical complications in cochlear implantation is the necrosis of the skin flap above the receiver-stimulator coil. We present a case of 55-year-old woman who underwent cochlear implantation and developed a bluish skin necrosis due to bone marking. The planned position for the receiver-stimulator was marked using methylene blue through skin to bone. She did not undergo skin flap thinning and underwent successful implantation with complete electrode insertion. Few weeks postoperatively, the patient developed bluish discoloration with progressive thick, blue eschar formation and skin flap necrosis. She subsequently underwent wound debridement and skin flap closure. Cochlear explantation was not necessary. Timely diagnosis and management about this complication is necessary to prevent further skin breakdown and subsequent device extrusion. This report identifies the marking using methylene blue as another possible source of skin flap necrosis in cochlear implantation, and surgeons should be aware of this potential complication.

J Audiol Otol 2015;19(2):108-110

KEY WORDS: Cochlear implantation · Methylene blue · Skin · Necrosis . Postoperative complications.

\section{Introduction}

Cochlear implants have been used to restore hearing in patients with severe to profound sensorineural hearing loss. Since cochlear implantation was introduced, many complications have been reported after the surgery. The complications were classified as either major or minor [1]. Major complications were those requiring surgical revision or resulting in a serious medical condition such as large scalp necrosis, severe infection, meningitis, and device failure [2]. Skin flap necrosis is one of serious complications because it results in exposure of the implant, thereby requiring explantation. Hematoma formation, infection, and wound dehiscence are common causes of flap necrosis [3]. Methylene blue dye has been widely used for the localization of the implant site. To date, no literature has reported complications after methylene blue dye injection. Herein, we report an unusual case of skin flap

This is an Open Access article distributed under the terms of the Creative Commons Attribution Non-Commercial License (http://creativecommons. org/licenses/by-nc/3.0/) which permits unrestricted non-commercial use, distribution, and reproduction in any medium, provided the original work is properly cited. necrosis due to bone marking with methylene blue after cochlear implantation.

\section{Case Report}

A 55-year-old woman with profound, sensorineural hearing loss underwent cochlear implantation with a Nucleus ${ }^{\circledR} 24$ contour (Cochlear Ltd., Sydney, Australia). She had a history of intracranial hemorrahge for which right pterional craniotomy was performed. Preoperative temporal bone MRI and CT scans were normal with the exception of microplate and screw in the right pterion. The planned position for the receiver-stimulator was marked using methylene blue. A sterile needle was inserted through the skin, until it touched the bone. $0.05 \mathrm{~mL}$ of methylene blue was then injected. She did not undergo skin flap thinning and underwent successful implantation with complete electrode insertion through the cochleostomy by way of the facial recess. The cochleostomy was sealed with a piece of muscle. The procedure was unenventful, and intraoperative blood loss was minimal. No adverse events were observed during the immediate postopera- 
tive period. However, 8 weeks after surgery, the woman presented with a bluish discoloration over the implant site, which was consistent with bone marking using methylene blue. She was otherwise well with no local erythema, fever, or swelling. The lesion developed a progressive thick, blue eschar formation and skin flap necrosis (Fig. 1). The flap necrosis did not respond to conservative treatment. There was

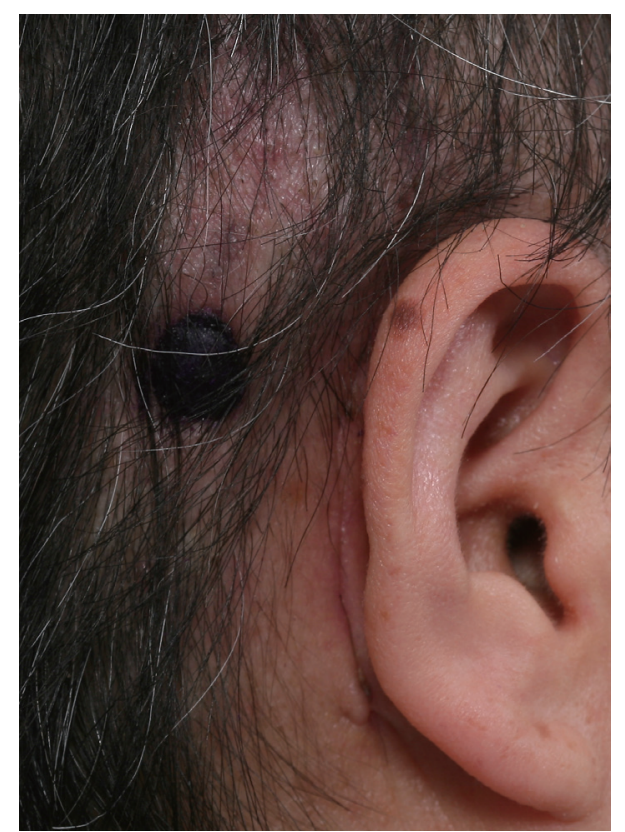

Fig. 1. Thick, blue eschar formation is seen in the postauricular region. The location is consistent with the site of bone marking done using methylene blue.

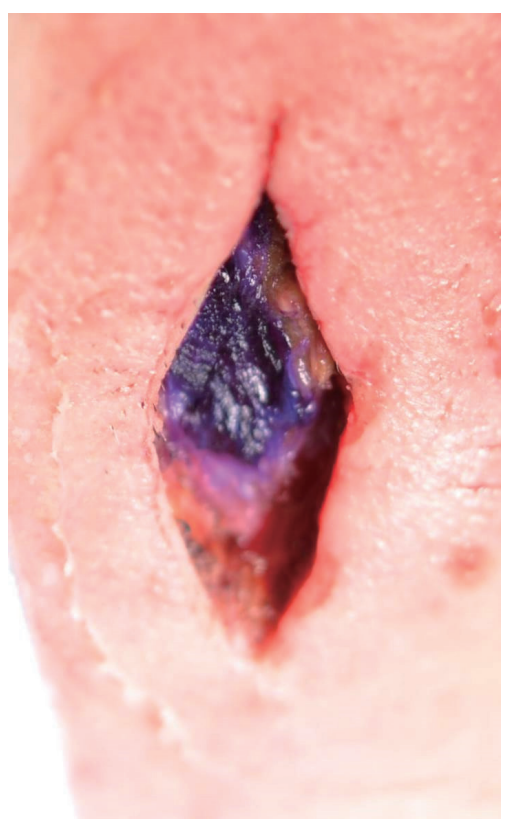

Fig. 2. Intraoperative finding after eschar removal and debridement. Bluish discoloration is seen in soft tissues over the implant site. no evidence of any electric or neural dysfunction. 11 weeks after surgery, she subsequently underwent wound debridement and skin flap closure; this procedure involved a linear skin incision made around the eschar in the postauricular region. A simple dissection allowed for removal and debridement of the eschar and the necrotic, discolored part of the skin and temporalis muscle (Fig. 2). The receiver-stimulator was identified and carefully preserved during removal of the eschar and soft tissues. The patient received intravenous antibiotics for 3 days and oral antibiotics for 2 weeks as a precautionary measure. The wound completely resolved after a month. The postoperative rehabilitation and implant function were excellent. During the 12-month follow-up, no recurrence was found.

\section{Discussion}

Cochlear implantation has been considered as a safe and reliable operation. However, major postoperative complications can develop in $1.5-8 \%$ of patients [4]. Skin flap necrosis is one of major complications requiring revision surgery. It can be caused by infection, hematoma, shape of the flap incision, or other abnormalities. Infection caused by various pathogens such as $P$. aeruginosa is a serious risk factor for flap necrosis. The pathogen can induce biofilm formation and result in recurrent infections, with the risk of implant extrusion [5]. Hematomas occur in the presence of coagulation disorders and inappropriate hemostasis. While this complication can be treated by re-intervention or aspiration, it may evolve into flap necrosis [6]. The technique for skin incision is a possible predisposing factor of flap necrosis. Smaller incisions with smaller skin flaps are used to reduce vascular compromise and minimize the risk of flap necrosis [7]. Thinner soft tissue and skin can also give the potential for flap breakdown. In addition, chronic pressure on the overlying skin such as using strong magnet can lead to flap necrosis. These are more likely to occur in children [8]. The management of skin flap necrosis is troublesome and sometimes needs multiple surgeries. It is most commonly treated using skin rotation flaps with device explantation [7,9]. This process has high morbidity and delays the use of the cochlear implant; hence, early recognition and treatment of this complication are important.

Methylene blue has been used extensively as a tool to localize the implant site in cochlear implantation. Methylene blue dye is a cationic thiazine that causes toxic effects and local inflammation by producing oxides and free radicals [10]. This dye also has vasoconstrictive effects by inhibiting the nitric oxide-mediated cyclic guanosine monophosphate path- 
way, which is responsible for smooth muscle relaxation and vasodilation [11]. Excessive use of this dye, particularly in tissues that are poorly perfused, may cause serious skin necrosis.

Moreover, intradermal injection of methylene blue has toxic effects on the local tissue and hence should be avoided [12]. Skin necrosis after the use of methylene blue dye has been reported in breast surgery. Described lesions range from localized blue staining, superficial ulceration and flap-site necrosis. Debridement and primary closure were performed for the treatemt of partial necrosis and wound dehiscence. However, the patients with wide skin necrosis underwent removal of necrotic tissue and implant, followed by musculocutaneous flap reconstruction $[10,12,13]$.

In the present case, the patient did not undergo skin flap thinning and did not use strong magnet. However, the patient had a history of neurologic surgery that might have caused poor tissue perfusion. Therefore, the flap necrosis might have developed due to the application of methylene blue to the site with poor local blood supply in the post-auricular area. During the surgery, blue-stained necrotic muscle and soft tissue were observed at the dye injection site. These findings were similar to those seen in the cases of skin necrosis by methylene blue toxicity after breast surgery [10]. Wound infections are typically characterized by fever, erythema, leukocytosis and purulent drainage and present rather quickly after surgery [14]. These characteristics were absent in our patient, and instead, a bluish discoloration developed 8 weeks after surgery; therefore we believe that the flap necrosis resulted from the toxic effects of methylene blue dye. Moreover, we cannot completely exclude the possibility of intradermal injection of the dye. In the present case, the onset of skin necrosis was relatively late compared with approximately 1 week in the cases of breast surgery. We believed that the early onset of skin necrosis in the cases of breast surgery results from larger amount of injection volume $(3-5 \mathrm{~mL})$ of the dye $[10,15]$.

When devascularization of tissues is expected such as revision surgery, prior surgery around temporal bone, thin skin flaps and excessive use of diathermy, the application of methylene blue as a bone marker is thought to be careful. Care should be taken to minimize skin complications by injecting the dye only above the bone and avoiding intradermal injection. When there is a suspicion of flap necrosis due to the methylene blue application, timely management is necessary to avoid progressive flap necrosis and implant extrusion.

We conclude that bone marking using methylene blue in- jection is another possible source of skin flap necrosis in cochlear implantation. Early diagnosis and management is necessary to prevent further skin breakdown and subsequent device extrusion.

\section{Acknowledgments}

This study was supported by research fund from Chosun University Hospital, 2015.

\section{REFERENCES}

1) Farinetti A, Ben Gharbia D, Mancini J, Roman S, Nicollas R, Triglia JM. Cochlear implant complications in 403 patients: comparative study of adults and children and review of the literature. Eur Ann Otorhinolaryngol Head Neck Dis 2014;131:177-82.

2) Qiu J, Chen Y, Tan P, Chen J, Han Y, Gao L, et al. Complications and clinical analysis of 416 consecutive cochlear implantations. Int J Pediatr Otorhinolaryngol 2011;75:1143-6.

3) Telian SA, El-Kashlan HK, Arts HA. Minimizing wound complications in cochlear implant surgery. Am J Otol 1999;20:331-4.

4) Tarkan Ö, Tuncer Ü, Özdemir S, Sürmelioğlu Ö, Çetik F, Kıroğlu $\mathrm{M}$, et al. Surgical and medical management for complications in 475 consecutive pediatric cochlear implantations. Int J Pediatr Otorhinolaryngol 2013;77:473-9.

5) Loeffler KA, Johnson TA, Burne RA, Antonelli PJ. Biofilm formation in an in vitro model of cochlear implants with removable magnets. Otolaryngol Head Neck Surg 2007;136:583-8.

6) Filipo R, D'Elia C, Covelli E, Bertoli GA, De Seta E, Manganaro F, et al. Haematoma after cochlear implantation: management of a minor complication. Acta Otolaryngol 2010;130:108-13.

7) Davids T, Ramsden JD, Gordon KA, James AL, Papsin BC. Soft tissue complications after small incision pediatric cochlear implantation. Laryngoscope 2009;119:980-3.

8) Das Purkayastha PK, Jewell S, James AL, Gordon K, Papsin B. Soft tissue complications after pediatric cochlear implantation in children younger than 12 months. Otol Neurotol 2011;32:780-3.

9) Cunningham CD 3rd, Slattery WH 3rd, Luxford WM. Postoperative infection in cochlear implant patients. Otolaryngol Head Neck Surg 2004;131:109-14.

10) Lee JH, Chang CH, Park CH, Kim JK. Methylene blue dye-induced skin necrosis in immediate breast reconstruction: evaluation and management. Arch Plast Surg 2014;41:258-63.

11) Dumbarton TC, Gorman SK, Minor S, Loubani O, White F, Green R. Local cutaneous necrosis secondary to a prolonged peripheral infusion of methylene blue in vasodilatory shock. Ann Pharmacother 2012;46:e6.

12) Stradling B, Aranha G, Gabram S. Adverse skin lesions after methylene blue injections for sentinel lymph node localization. Am J Surg 2002;184:350-2.

13) Reyes F, Noelck M, Valentino C, Grasso-Lebeau L, Lang J. Complications of methylene blue dye in breast surgery: case reports and review of the literature. J Cancer 2010;2:20-5.

14) Loochtan MJ, Yang S, Mantravadi AV, Marzo SJ. Cochlear implant extrusion secondary to keloid formation. Cochlear Implants Int 2014; 15:276-8.

15) Bleicher RJ, Kloth DD, Robinson D, Axelrod P. Inflammatory cutaneous adverse effects of methylene blue dye injection for lymphatic mapping/sentinel lymphadenectomy. J Surg Oncol 2009;99: $356-60$. 